Ciência e Natura, v. 37 Part 2 2015, p. 190-194 ciênciaenatura

\title{
Adjust genetic algorithm parameter by fuzzy system
}

Tayebe Noshadi ${ }^{1}$, Marzieh Dadvar2 ${ }^{2}$ Nastaran Mirza ${ }^{3}$, Shima Shamseddini ${ }^{4}$

${ }^{1}$ Student of Master Course, artificial intelligence, Islamic Azad University, Science and Research

Branch, Bushehr

${ }_{2}^{2}$ Phd student, artificial intelligence, Islamic Azad University, Science and Research Branch, Tehran

${ }^{3}$ Student of Master Course, artificial intelligence, Islamic Azad University, Science and Research

Branch, Bushehr

${ }^{4}$ Student of Master Course, artificial intelligence, Islamic Azad University, Science and Research

Branch, Bushehr

\begin{abstract}
Genetic algorithm is one of the random searches algorithm. Genetic algorithm is a method that uses genetic evolution as a model of problem solving. Genetic algorithm for selecting the best population, but the choices are not as heuristic information to be used in specific issues. In order to obtain optimal solutions and efficient use of fuzzy systems with heuristic rules that we would aim to increase the efficiency of parallel genetic algorithms using fuzzy logic immigration, which in fact do this by optimizing the parameters compared with the use of fuzzy system is done.
\end{abstract}

Keywords: genetic algorithms, fuzzy systems, genetic algorithms, parallel, parallel genetic algorithm immigration 


\section{Introduction}

characteristic of a good gene breeding a new generation [1] and in solving complex problems, simple GA do not work well, but the field of genetic algorithms called parallel genetic algorithm to solve the problem of time-consuming and complex, easier to work and reduces costs and to prevent sudden convergence and sharing high-quality solution method) (MPGA that we use, consists different models and eventually parallel genetic algorithm immigration

\section{Genetic Algorithm (GA)}

Since the early 1950s, attempts to simulate the phenomenon of human evolution began for computers among many researchers in the field of mathematical sciences and engineering were attracted to this area. Finally, in the early 1970s, John Holland (John Holland), in his book introduced the GA as a tool to optimize public. GA is a special kind of evolutionary algorithms derived from natural evolution and genetic in nature.

A disciple of John Holland, David Goldberg sporadic tasks that had been raised by the Holland with the results of own research, published in the form of a comprehensive book. It can be said Goldberg, with the publication of the book has contributed most to the development and introduction of genetic algorithms. These Algorithms are powerful search methods that are used to solve different problems in all areas.

A - The genetic algorithm steps

- Create random population and assessment of (population)

- Parental choice and combine them to create a population of children (crossover)

- Select members of the crowd for mutation (mutation)
- Combination (merger) of the population, children and mutants and create new original population

- If the condition is not fulfilled end of step 2 again there where

- End

B- The end conditions

- achieve an acceptable level of response

- Elapsed time / repeat certain

- Elapsed time / frequency of occurrence of certain specific improvements without result

- reaching a specified number of NFE

GA by roulette wheels or race, select the best of the population [1]. Although GAs are not very complicated in terms of performance but are costly and time-consuming algorithm. In this algorithm, the larger and more complex the problem, the program will increase its costs.

Evaluate people by sending a fraction of the population to any available processor, parallelism occurs. Relationship shown when each of the agents are all individuals assess their fitness values return to the original community.

\section{Parallel Genetic Algorithm (PGA)}

Parallel implementation of a simple genetic algorithm, called parallel genetic algorithm. [3]

This method in some problems, including the problem-solving task scheduler is used as the solution of this problem by applying different constraints and different priority optimization can be achieved. PGA because the issues raised by the GA simple, to find the optimal solution in the search space is very large, very long execution time needed by the PGA, less time to reach the optimal solution. In addition, PGA has significant 
advantages in terms of scalability and performance. PGA simply heterogeneous computers on a network or on a parallel supercomputer to be implemented.

GA, which simply means it can be parallelized related to the following:

- In what ways competency assessment solutions and mutation are applied,

- How to select the operator to apply, for example, local or national selection (the sample)

- Have one or several sub-population of the community is used (the interaction between the community),

- If several sub-population used how solutions can be exchanged among them.

PGA has different methods as follows:

- independent PGA

- immigration PGA

- zoned PGA

- segmented PGA

- immigration and segmented PGA

\section{Migration Parallel Genetic Algorithm (MPGA)}

The method \#2 used here. This method involves adding the periodic migration of chromosomes among processing elements by independent PGA. The purpose of this procedure is to prevent the sudden convergence and share solutions with high quality technology. Migration of chromosomes occurs after a certain number of repetitions. Each processing element has its own local copy of the chromosome to the processing element number 1 to number $n$ to the first step of sending immigrants, then the second step of the migration of $\mathrm{n}$ to send to the processing element (2) and so on. Chromosomal that received the worst alternative local chromosome unless a chromosome as the chromosome exist below the entry.
PGA introduced the following two methods use virgin:

- Standard parallel method

- Decomposition method (Decomposition Approach)

In the analysis of a number of subpopulations in the name of the community to break down. Sectors (as part of the country) are separated and people are fully within a section. The operator added that migration called for the movement of people from one section to the other sections of this operator is used. If people can migrate from one part to another part of the island is called. Migration can be controlled with different parameters such as the rate of immigration, the structure of immigration, like worst, best and random immigration.

Note: The four models have been proposed to implement parallel genetic algorithms:

1. The slave / master single global population

2. Single population model grainer-fine

\section{Multi population}

\section{Hierarchical models}

Here we use master/slave method for implementation. The algorithm used in this method only gives a society and just assessment of parallel conduct, or the genetic operators and evaluation of individuals is done in parallel. And this method does not affect the behavior of the GA.

\section{Fuzzy system}

Fuzzy systems, are knowledge based systems and their rules. The heart of a fuzzy system is a knowledge database that consists of fuzzy IF-THEN rules. An if-then fuzzy rule is an if-then statement that some words are marked by continuous membership functions [2]. In this article we use Mamdani fuzzy system to regulate and control the GA parameters to improve performance. 
When we use a fuzzy system as a controller then called fuzzy controller. A control problem is designing fuzzy controller that produce the best objectives. These objectives include:

1.The pursuit of well-being

2. The lack of effect of noise and distortion in the output

3. low sensitivity to changes in process control output

4. The stability of the system under different conditions

then briefly explain Mamdani fuzzy system

\subsection{Mamdani fuzzy system}

in 1975, Mamdani and Asylyan determined a basic framework for the fuzzy controller applied it to a steam engine [2]. They found that the construction a fuzzy controller is very simple and works well. Here we are referring to the Mamdani fuzzy system that is used in this experiment. In this model, the rules are as follows:

If $\mathrm{x}$ is $\mathrm{A} 1$ and $\mathrm{y}$ is $\mathrm{B} 1$ then $\mathrm{z}$ is $\mathrm{C} 1$

$$
\text { If } x \text { is } A 2 \text { and } y \text { is } B 2 \text { then } z \text { is } C 2
$$

For example, if the room temperature is "slightly high" and humidity is "quite a lot". Then the air conditioning set on "high or high" position.

5. The Fuzzy Migration Parallel Genetic Algorithm (FMPGA)

in PGA algorithms, cutting rate and mutation rate specified by the user, but in this article these rates are produced by the fuzzy system each time that this method significantly increases the efficiency of PGA algorithms. The input fuzzy systems is as follows:

$$
\begin{aligned}
& f_{\text {ave }}=\frac{\text { sum of all } f}{\text { number }} \\
& \text { norm } f_{\text {ave }}=\frac{f_{\text {ave }}-f_{\text {max }}}{f_{\text {min }}-f_{\max }}
\end{aligned}
$$

norm $=\frac{f_{\text {min }}-f_{\text {ave }}}{f_{\min }-f_{\text {max }}}$

That:

(fitness) $f$

$f_{\text {ave }}$ (Average $\left.f\right)$

$f_{\min }($ Worst $f)$

$f_{\max }($ Best $f)$

That it's output is mutation rate.

\section{Test:}

We have done a test, using the PGA, consider 1000 population that is divided into a scale of 100. This indicates the presence of 100 genes per chromosome.

Our population is divided into 5 sub-200 respectively. By MPGA the best solution of all subpopulations migrate to neighboring subpopulations. (Ring topology)

FPGA parameters using fuzzy rules regulating the PGA subpopulations that Mamdani fuzzy laws have been used here (that is, the mutation parameters are fuzzy and fuzzy cut). In this way we archive of the chromosomes $(5 \%)$ of the subpopulations that are stored in each time you run make. When the archive is filled this population replace as main population again. And in this system we use Mamdani system

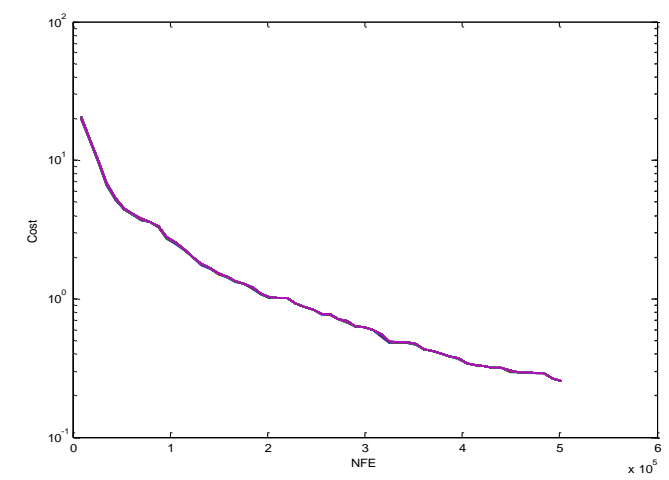

Figure 1: the above chart has been drawn for total population 1000 which is divided to 5 subpopulation 200 that $\mathrm{NFE}=500500$ in BestCost $=0.2485$ 
Bestcost and NFE After returning from the sub-populations were drawn in the array.

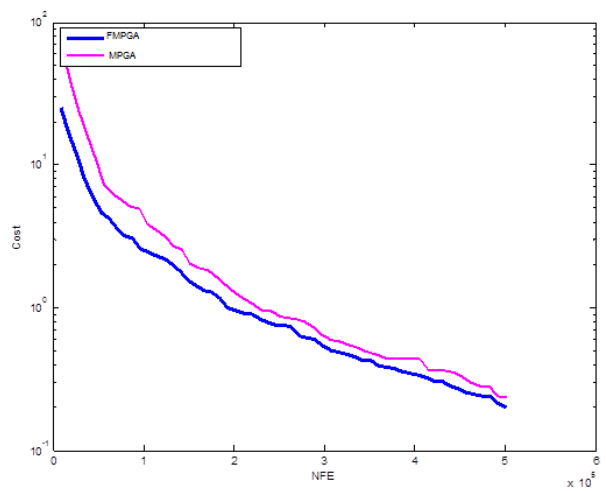

Figure 2: In this diagram, MPGA shown with purple and FMPGA shown with blue.

\section{7 conclusion}

in this paper we did an experiment to optimize the GA parameters using fuzzy system. Mutations in the genetic algorithm parameters specified by the user, but in our tests these parameters are determined each time by the fuzzy system and the results show that the parameters that have been set by FMPGA algorithm is better than parameter that produced by MPGA in quality and performance. In fact, although in MPGA the best answer migrate from the neighboring populations and have good results, but, in FMPGA method, before migration the best answers to the neighboring populations by FPGA, all parameters optimized through fuzzy system (Mamdani). We try to optimize GA parametersalso, offer new ways to choose the best population replacement rates

Table 1: Dimention=100, Population Size $=1000$

\begin{tabular}{|c|c|c|c|c|}
\hline Function & PGA & $\begin{array}{c}\text { PGA } \\
\text { Migration }\end{array}$ & Fuzzy PGA & $\begin{array}{c}\text { Fuzzy PGA } \\
\text { Migration }\end{array}$ \\
\hline $\begin{array}{l}\text { De Jong's } \\
\text { (Nfe) } \\
\text { (Exchange } \\
\text { Population) }\end{array}$ & $\begin{array}{c}0 . \leqslant \leq r q \\
500500 \\
2\end{array}$ & $\begin{array}{c}0.24854 \\
500500 \\
2\end{array}$ & $\begin{array}{c}0 . \text { โ YAV } \\
500072 \\
2\end{array}$ & $\begin{array}{c}0.23764 \\
500200 \\
2\end{array}$ \\
\hline
\end{tabular}

\section{References:}

1. Soung-Min Im and Ju-Jang Lee, Adaptive Crossover," Mutation and Selection Using Fuzzy system for Genetic Algorithms", Electrical Engineering and Computer science korea advanced institute of science and technology (KAISI) 373-1, Guseongdong,yuseong-gu, Daejeon, 305-701, korea

2. Li.Wang,(1962)" Fuzzy systems, fuzzy control", K.N.Toosi University of Technology, Eighth Edition, October 1392.

3. S.N. Deepa, Introduction to Genetic Algorithms, 2008) 